OPEN ACCESS

Edited by:

Angel Lanas,

University of Zaragoza, Spain

Reviewed by:

Francisco Schlottmann

Hospital Alemán, Argentina

Vivek Kaul,

University of Rochester, United States

${ }^{*}$ Correspondence:

Atul Maini

Atul_Shyla@yahoo.com

Specialty section: This article was submitted to

Gastroenterology,

a section of the journal

Frontiers in Medicine

Received: 31 January 2021

Accepted: 05 October 2021

Published: 10 November 2021

Citation:

Maini A, Sun J, Buniak B, Jantsch S, Czajak R, Frey T, Kumar BS and

Chawla A (2021) Heartburn Center

Set-Up in a Community Setting:

Engineering and Execution.

Front. Med. 8:662007.

doi: 10.3389/fmed.2021.662007

\section{Heartburn Center Set-Up in a Community Setting: Engineering and Execution}

\author{
Atul Maini ${ }^{1 *}$, John Sun ${ }^{1}$, Borys Buniak ${ }^{1}$, Stacey Jantsch ${ }^{1}$, Rachel Czajak ${ }^{1}$, Tara Frey ${ }^{1}$, \\ B. Siva Kumar ${ }^{1}$ and Amarpreet Chawla ${ }^{2}$ \\ ${ }^{1}$ The Heartburn Center at St. Joseph's Health, Liverpool, NY, United States, ${ }^{2}$ Johns Hopkins University, Baltimore, MD, \\ United States
}

Background: Optimal management of gastroesophageal reflux disease (GERD) requires a concerted team of physicians rather than an individual approach. While an integrated approach to GERD has previously been proposed, the practical execution of such a "center of excellence" (COE) has not been described, particularly in a community setting. Ranging from initial consultation and diagnosis to surgical intervention for complex disease, such an approach is likely to provide optimal care and provide surveillance for patients with a complex disease process of GERD.

Methods: We report our approach to implement an integrated heartburn center $(\mathrm{HBC})$ and our experience with the first cohort of patients. Patients treated in the HBC were followed for 2 years from initial consultation to completion of their appropriate treatment plan, including anti-reflux surgery. The performance prior to the HBC set-up was compared to that post-HBC. Performance was measured in terms of volume of patients referred, referral patterns, length of stay (LOS), and patient health-related quality of life (HRQL) pre- and post-surgery.

Results: Setting up the HBC resulted in referrals from multiple avenues, including primary care physicians (PCPs), emergency departments (EDs), and gastroenterologists (Gls). There was a 75\% increase in referrals compared to pre-center patient volumes. Among the initial cohort of 832 patients presenting to the HBC, $<10 \%$ had GERD for $<1$ year, $\sim 60 \%$ had GERD for $1-11$ years, and $~ 30 \%$ had GERD for $\geq 12$ years. More than one-quarter had atypical GERD symptoms (27.6\%). Only $6.4 \%$ had been on PPIs for $<1$ year and $>20 \%$ had been on PPIs for $\geq 12$ years. Thirty-eight patients were found to have Barrett's esophagus (4.6\%) (up to 10 times the general population prevalence). Two patients had dysplasia. Seven patients $(0.8 \%)$ received radiofrequency ablation (RFA) for Barrett's esophagus and two patients received endoscopic mucosal resection (EMR) for Barrett's esophagus-related dysplasia. The most common comorbidities were chronic pulmonary disease (16.8\%) and diabetes without complications (10.6\%). Patients received treatment for newly identified comorbid conditions, including early maladaptive schemas (EMS) and generalized anxiety disorder (GAD) $(n=7 ; 0.8 \%)$. Fifty cases required consultation with various specialists $(6.0 \%)$ and 34 of those $(4.1 \%)$ resulted in changes in care. Despite the significant increase in patient referrals, conversion rates from diagnosis to anti-reflux surgery remained consistent at $~ 25 \%$. Overall HRQL improved 
year-over-year, and LOS was significantly reduced with potential cost savings for the larger institution.

Conclusions: While centralization of GERD care is known to improve outcomes, in this case study we demonstrated the clinical success and commercial viability of centralizing GERD care in a community setting. The integrated GERD service line center offered a comprehensive, multi-specialty, and coordinated patient-centered approach. The approach is reproducible and may allow hospitals to set up their own heartburn COEs, strengthening patient-community relationships and establishing scientific and clinical GERD leadership.

Keywords: gastroesophageal reflux disease (GERD), center of excellence (CoE), heartburn center (HBC), quality of life (HRQL), length of stay (LOS)

\section{INTRODUCTION}

Gastroesophageal reflux disease (GERD) is a complex disease process that affects $\sim 20 \%$ of adults in the US $(1,2)$. Many patients with GERD self-medicate or are treated with medication for years without proper work-up or evaluation by a physician. Patients with persistent and intense symptoms of GERD (i.e., refractory GERD) experience decreased healthrelated quality of life (HRQL) and lower productivity at work and in daily activities, potentially resulting in higher healthcare and employer costs (3). Persistent reflux symptoms, despite medical therapy, have been associated with debilitating psychological comorbidities including mental health disorders, sleep disorders, and psychological distress to patients (4-6). GERD has also been shown to be associated with medical comorbidities including hypertension, diabetes, hyperlipidemia, coronary heart disease, alcohol-related illness, stroke, obesity, chronic obstructive pulmonary disease, asthma, biliary stone, anxiety, depression, chronic kidney disease, and cirrhosis (7, 8). GERD can also have significant potential complications such as dysphagia, erosive esophagitis, Barrett's esophagus, and esophageal adenocarcinoma (9).

The effective treatment of GERD patients requires an awareness of the clinical spectrum of GERD and its varied symptomatology, associated comorbidities, and potential complications. Despite the prevalence and impact of GERD, the patient and treatment pathways for a considerable number of patients with GERD are suboptimal $(10,11)$. Patients with GERD may present with typical and/or atypical symptoms that may be missed if not appropriately recognized, diagnosed, and treated. Due to the association of GERD with many psychological and medical comorbid conditions, screening and treatment of GERD may aid as a predictor and allow for earlier detection and treatment of undiagnosed comorbid diseases. However, referral pathways for patients with GERD from primary care physicians (PCPs) to specialists are fragmented and not adequately organized, thus affecting the process of patient evaluation, treatment, continuity of care, and ultimately clinical outcomes and costs (12).

Centers of excellence (CoEs) are specialized programs that supply high concentrations of expertise and related resources centered on particular medical areas and delivered in a comprehensive, interdisciplinary fashion (13). CoEs provide an opportunity to align physicians in quality improvement, reduce costs through greater efficiencies, and create market differentiation through clinical excellence and high patient satisfaction (14-18). CoEs also have the ability to dramatically enhance the depth and breadth of healthcare services available in communities that otherwise would not have had access to such exceptionally high levels of quality care (13).

In order to optimize treatment options for GERD, the concept of a "Heartburn Center" as a CoE in foregut disease is emerging as our understanding of the management of the array of esophageal diseases continues to grow (19). An integrated and collaborative multi-specialty approach to the management of referral, patient, and treatment pathways is designed to provide a comprehensive pathway of care for GERD by improving communication, education, and compliance with testing and treatment. A CoE approach has been associated with improved healthcare economics and patient outcomes $(1,20)$.

While significant strides have been made in defining the quality of GERD care in an integrated CoE (19), the question remains as to what the underlying components in successfully executing those best practices in such a center would be. As such, the purpose of this study was to describe our approach to implement a heartburn center (HBC) at our communitybased facility, report potential challenges and barriers in setting up and running these centers and steps to mitigate them, and most importantly, provide a reproducible methodology for the design and development of a functional HBC with the aim to have hospital administrators and healthcare professionals identify practices, tools, and resources needed to set up their comprehensive heartburn CoE.

\section{MATERIALS AND METHODS}

The concept of the interdisciplinary practice model was first introduced to our hospital leadership team in early 2016 by a surgeon and GI. St. Joseph's Physicians Surgical Services, part of St. Joseph's Hospital Health Center, offers comprehensive surgical care for ambulatory, elective and critically ill patients. 
Surgical services range from oncology, bariatric, including both laparoscopic and robotic surgeries. As illustrated in Figure 1, a successful heartburn $\mathrm{CoE}$ is supported by three key elements: a team of healthcare providers, hospital administration, and referral sources.

\section{Referral Sources}

To provide optimal patient care, it is critical that evidence-based multi-disciplinary referral pathways are set up that include but are not limited to patients, PCPs, GIs, ear, nose, and throat physicians (ENTs), pulmonary physicians, emergency physicians, and surgeons. Figure 2 demonstrates the integrated organization structure in place at our HBC.

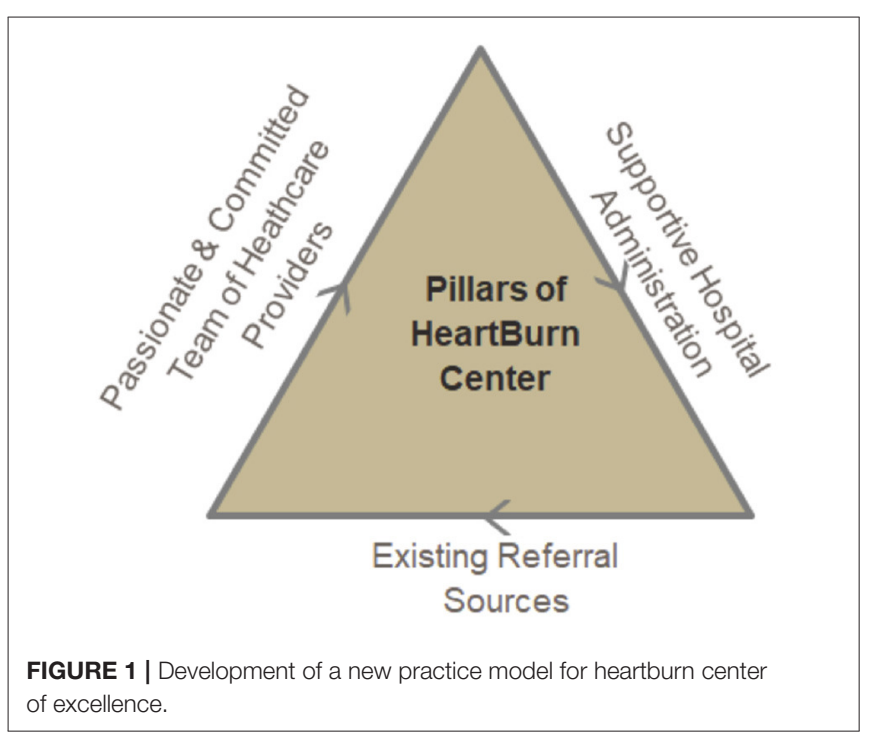

GIs should be a part of the core group and should be involved early in the patient's treatment decision. A previous administrative claims database analysis that examined healthcare resource utilization of patients with a diagnosis of GERD found that esophageal objective testing was inadequate when patients were not referred to GIs, and furthermore, timely transfer of GERD patients to GIs when empiric treatment is insufficient may lead to improved clinical management (21). It is our estimate that in our $\mathrm{HBC}$, as many as $20 \%$ of patient referrals may have been lost if GIs were not integrated into the HBC. However, to ensure timely referrals and to avoid referrals from GIs that are only limited to those patients with long-term, severe symptoms and large hiatal hernias, improved education for recognizing symptoms suggestive of GERD, and consequently to appropriately channel patients through diagnostic and treatment pathways (22).

\section{Hospital Administration}

An optimized program has well-coordinated milestones of timely consultation, diagnostic workup, a documented treatment plan, and regular follow-up.

\section{Consultation}

For patients to be funneled into the center, we developed a patient outreach program that facilitated patient flow, importantly with dedicated program coordinator and director. Such a program ensured patient education about the center's value proposition and differentiation from other similar centers in the region. Centers may consider developing a hub to receive dedicated calls from potential patients and appropriate referrals. Following referral, it is critical to set outcome expectations with the patient and expectations of downstream effect.

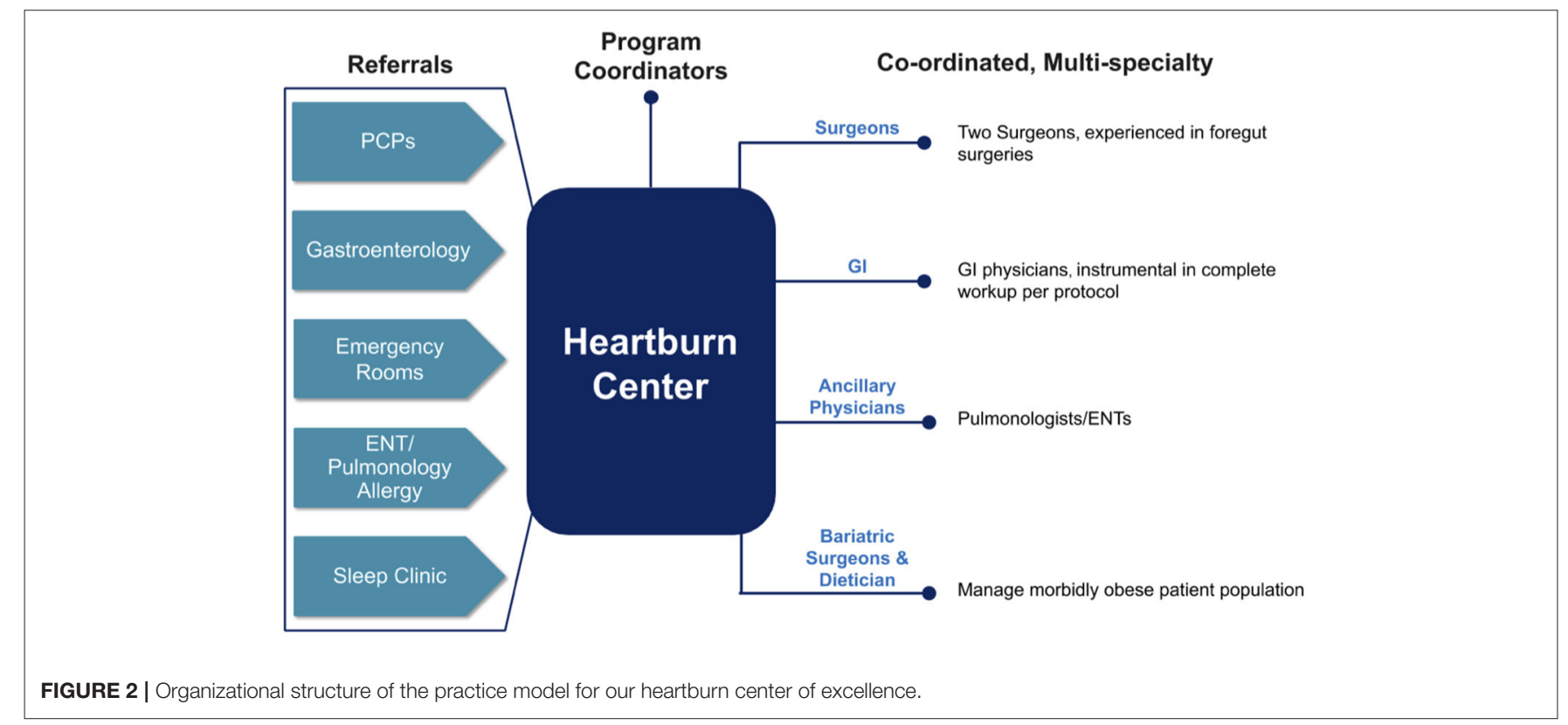




\section{Patient Navigation Platform}

A digital platform that facilitates patient progress tracking throughout their continuum of care and also facilitates comprehensive data collection regarding the effectiveness of such a center is vital for a service line center. An integrated implementation of such a platform with the Epic electronic health record (Epic Corporation, Madison, WI) system at our center facilitated referrals from primary care and ED. Especially patients who came to the ED with chest pain and who were typically sent back if no cardiac involvement was determined, are now being regularly referred to the $\mathrm{HBC}$ with improved disease awareness of atypical symptoms of GERD as well as the facility to seamlessly refer those patients to the HBC.

\section{Diagnostic Work-Up}

Having a protocol for appropriate identification of patients is important. Esophageal objective testing is required to anatomically and physiologically evaluate the presence and progression of GERD in patients being considered for surgery. Founded on evidence- and experienced-based consensus $(23,24)$, we established a robust diagnostic protocol that included endoscopy, ambulatory $\mathrm{pH}$ monitoring, manometry, and evaluation of LES length and degree of hiatal herniation by EGD for evaluating the patient's condition and to rule out other diagnoses such as malignancies, achalasia, and scleroderma (25). At our center, we combined the results of all the diagnostics to document the presence and extent of the disease and assist in planning the surgical approach.

\section{Treatment Plan}

We set up a treatment protocol for every patient prior to the start of disease management. We have described the selection criteria in our manuscript which is based on patients Symptoms, EMS study, pH study, Endoscopy, Barium swallow study and GAD-Generalized Anxiety score and patient preference. Our protocol dictates that post-diagnostic workup, the patient be offered treatments based on the pathology and to rule out other intra-abdominal pathologies such as gallbladder or peptic ulcer disease that may be potentially misdiagnosed as reflux disease. Patients who are candidates for anti-reflux surgery are given options to undergo either a hiatal hernia repair with magnetic sphincter augmentation device implant/LINX ${ }^{\circledR}$ procedure or a robotic hiatal hernia procedure with a posterior robotic Toupet fundoplication (RTF). Patients with a BMI > 35 are first educated about lifestyle modification including weight loss or referred to a bariatric surgeon if surgery is indicated. Patients with any anxiety causing esophageal symptoms are referred to a gut psychologist. Importantly, recurring clinical meetings and grand rounds are setup with core physicians to discuss an optimal treatment plan for each patient.

\section{Follow-Up}

The patients in the center are typically followed at 2, 8 weeks, 6 months and 1 year in the postoperative period in patients with LINX ${ }^{\circledR}$ procedure and fundoplications. The patients are surveyed and their HRQL scores are calculated at each visit.

\section{Team of Healthcare Providers}

Central to the HBC is the surgeon and GI partners. It is critical that the surgeons have foregut surgery experience and that the GIs are experienced in foregut physiology and pathology and are willing to follow established pre-surgery diagnostic pathways including manometry and $\mathrm{pH}$ testing, understand the US payer environment, and importantly have demonstrated history of collaborating with administration and colleagues.

Ancillary physicians may include a gut psychologist, pulmonologist, ENT specialists, bariatric surgeon, and nutritionist. A supportive staff of healthcare providers should include a program coordinator, nurse practitioners, physician assistants, and a nutritionist. Finally, it is important to partner with the hospital administration such as a program director who has the authority to approve new programs or looking to establish leadership in a particular disease area.

\section{HBC Performance Measurement: Methodology}

We followed patients over 2 years from initial consultation to completion of an appropriate treatment plan. Figures 3A,B illustrates the patient flow protocol and treatment decision tree that were implemented at our $\mathrm{HBC}$, respectively. Retrospective data at each node of patients' treatment pathway were collected and analyzed. Specifically, four endpoints were assessed and quantified: (1) Patient volumes and corresponding conversion rates through consult-diagnostics-treatment; (2) Referral patterns and sources of referral; (3) Trends on length of stay, and finally; (4) Patient outcomes in terms of HRQL impact.

\section{Inclusion Criteria}

The study included patients who were 21 years of age and older, had a history of typical or atypical GERD symptoms, at least 8 weeks of once or twice daily PPI therapy with or without breakthrough symptoms, actively seeking alternative surgical treatment options for bothersome GERD symptoms including, but not limited to heartburn, regurgitation, and dysphagia. Subjects were willing and able to cooperate with follow-up examinations. For those having LINX $^{\circledR}$ implanted, the LINX ${ }^{\circledR}$ Instruction for Use (IFU) (26) was additionally followed as the inclusion criteria.

\section{Exclusion Criteria}

Subjects with suspected or known allergies to titanium, stainless steel, nickel, or ferrous materials, esophageal dysmotility, connective tissue disorders, or severe anxiety symptoms.

\section{RESULTS}

\section{HBC Assessment and Metrics}

Tables 1, 2 present the baseline demographic and clinical characteristics of the first 832 patients who presented to the HBC. Approximately one-third of patients were younger than 45 , onefifth were older than 65 , and $45.2 \%$ were between 46 and 65 years of age. Six out of 10 patients were female and most (83.1\%) were Caucasian (7.1\% Black or African American, 5.0\% other, 3.1\% Hispanic, and $1.7 \%$ Asian). Half of the patients had commercial 
A

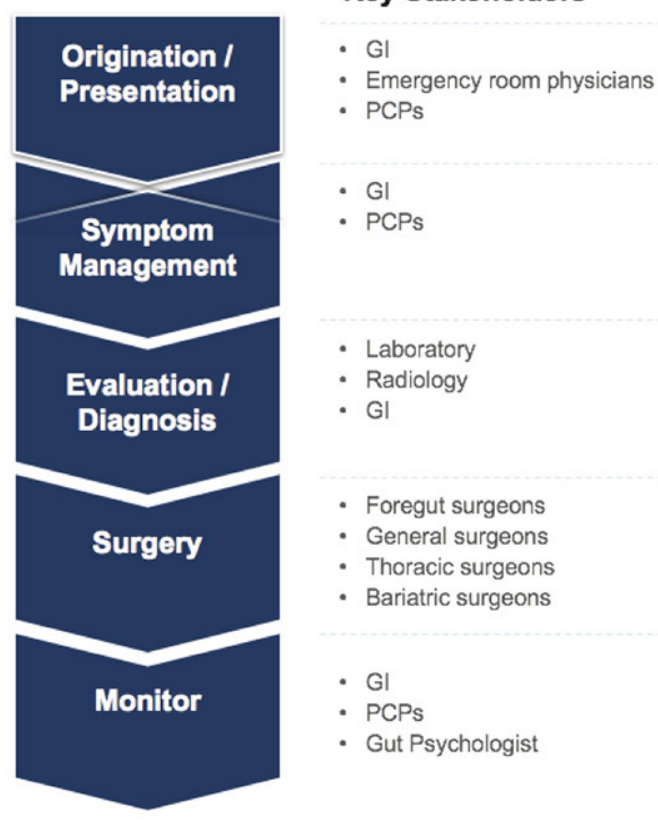

B

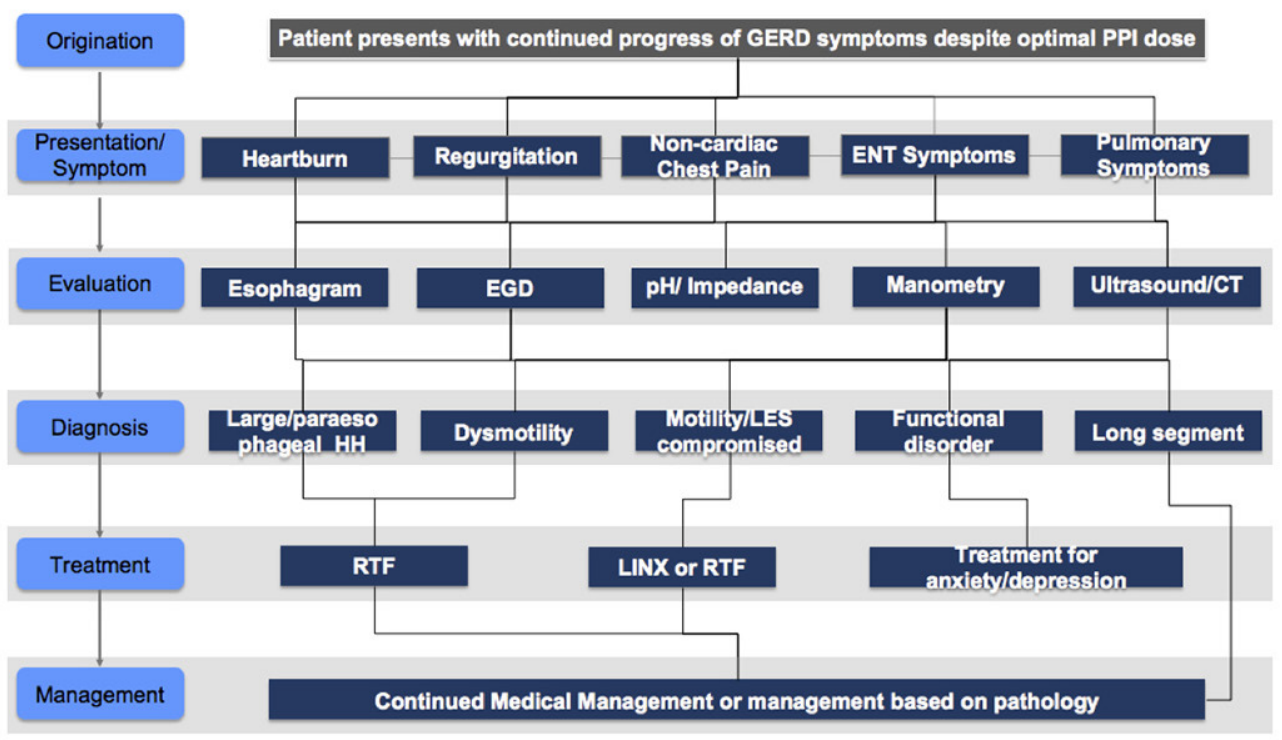

FIGURE 3 | (A) Patient flow and associated providers. (B) Treatment decision tree implemented at our heartburn center.

insurance $(49.4 \%)$ and the remainder had Medicaid $(28.2 \%)$ or Medicare (22.4\%).

Approximately one in five patients had normal weight, onethird were overweight, and the remainder (46.5\%) were obese (Table 2). More than half of patients had a Charlson Comorbidity Index (CCI) score of 0 , nearly one-third had a CCI of 1-2, nearly $10 \%$ had a score of $3-4$, and only $2.2 \%$ had a score of 5 or greater. The most common comorbidities were chronic pulmonary disease $(16.8 \%)$ and diabetes without complications
(10.6\%) (Table 2). Patients received treatment for any newly identified comorbid conditions, including early maladaptive schemas (EMS) and generalized anxiety disorder (GAD) $(n=$ $7 ; 0.8 \%$ ) (data not shown). In total, 50 of the 832 cases required conference/consultation with various specialists (6.0\%) and 34 of those $(4.1 \%)$ resulted in changes in care. Seventeen of the cases (3.0\%) resulted in changes in medical management and 17 (3.0\%) resulted in a change in the type of recommended GERD surgery (data not shown). 
TABLE 1 | Baseline demographic characteristics of patients presenting to the HBC.

\begin{tabular}{|c|c|c|}
\hline $\begin{array}{l}\text { Patient demographic } \\
\text { characteristic/comorbidity }\end{array}$ & $N$ & $\% /$ mean (SD) \\
\hline All & 832 & $100 \%$ \\
\hline \multicolumn{3}{|l|}{ Age } \\
\hline$<19$ & 2 & $0.2 \%$ \\
\hline 19 to 25 & 39 & $4.7 \%$ \\
\hline 26 to 45 & 247 & $29.7 \%$ \\
\hline 46 to 65 & 378 & $45.4 \%$ \\
\hline$>65$ & 166 & $20.0 \%$ \\
\hline \multicolumn{3}{|l|}{ Gender } \\
\hline Female & 500 & $60.1 \%$ \\
\hline Male & 332 & $39.9 \%$ \\
\hline \multicolumn{3}{|l|}{ Race } \\
\hline Caucasian & 691 & $83.1 \%$ \\
\hline Black or African American & 59 & $7.1 \%$ \\
\hline Asian & 14 & $1.7 \%$ \\
\hline Hispanic & 26 & $3.1 \%$ \\
\hline American Indian/Alaska Native & 0 & $0.0 \%$ \\
\hline Other & 42 & $5.0 \%$ \\
\hline Unknown/Refused & 0 & $0.0 \%$ \\
\hline \multicolumn{3}{|l|}{ Health insurance payer } \\
\hline Medicaid/Molina/Fidelis/Government plans & 235 & $28.2 \%$ \\
\hline Commercial & 411 & $49.4 \%$ \\
\hline Medicare & 186 & $22.4 \%$ \\
\hline
\end{tabular}

More than one-quarter of patients with GERD had atypical symptoms (27.6\%). $<10 \%$ of patients had GERD for $<1$ year, $\sim 60 \%$ had GERD for $1-11$ years, and 3 in 10 patients had GERD for 12 or more years. Only $6.4 \%$ of patients had been on PPIs for less than a year and more than $20 \%$ of patients had been on PPIs for 12 or more years. Thirty-eight patients had Barrett's esophagus (4.6\%) (Table 2), which is up to 10 times the prevalence rate found in the general population (27). Two patients were found to have dysplasia. Seven patients $(0.8 \%)$ received radiofrequency ablation (RFA) for Barrett's esophagus and two patients received endoscopic mucosal resection (EMR) for Barrett's esophagus-related dysplasia (data not shown).

Trends in the performance of the HBC were assessed over 2 years, specifically 2017-2019, and benchmarked across four different metrics: referrals, patient volumes, length of stay, and HRQL.

\section{Referrals Patterns and Sources}

The primary source of referrals were self-referrals (patients from advertisements, television events, print, public patient seminars (24\%), GI (20\%), primary care centers (54\%), with others from emergency department (ED) and specialty services. Most notably, GIs were a de novo referral source that were unavailable to our institution before the setup of the HBC.

These patients were distributed across 79 zip codes around the HBC. Although there are hospitals in this catchment area
TABLE 2 | Baseline clinical characteristics of patients presenting to the HBC.

\begin{tabular}{|c|c|c|}
\hline $\begin{array}{l}\text { Patient demographic } \\
\text { Characteristic/Comorbidity }\end{array}$ & $N$ & $\% /$ mean (SD) \\
\hline All & 832 & $100 \%$ \\
\hline \multicolumn{3}{|l|}{ Body mass index (BMI) } \\
\hline <18.5 (Underweight) & 6 & $0.7 \%$ \\
\hline 18.5-24.9 (Normal) & 165 & $20.2 \%$ \\
\hline 25.0-29.9 (Overweight) & 266 & $32.6 \%$ \\
\hline 30.0-<35.0 (Obesity Class I) & 219 & $26.8 \%$ \\
\hline 35.0-<40.0 (Obesity Class II) & 99 & $12.1 \%$ \\
\hline 40.0+ (Obesity Class III) & 62 & $7.6 \%$ \\
\hline \multicolumn{3}{|l|}{ Charlson comorbidity score } \\
\hline 0 & 469 & $56.4 \%$ \\
\hline $1-2$ & 265 & $31.9 \%$ \\
\hline $3-4$ & 80 & $9.6 \%$ \\
\hline$\geq 5$ & 18 & $2.2 \%$ \\
\hline \multicolumn{3}{|l|}{ Comorbidities } \\
\hline Myocardial infarction & 40 & $4.8 \%$ \\
\hline Congestive heart failure & 12 & $1.4 \%$ \\
\hline Peripheral vascular disease & 35 & $4.2 \%$ \\
\hline Cerebrovascular disease & 32 & $3.8 \%$ \\
\hline Chronic pulmonary disease & 140 & $16.8 \%$ \\
\hline Connective tissue disease-rheumatic disease & 38 & $4.6 \%$ \\
\hline Peptic ulcer disease & 15 & $1.8 \%$ \\
\hline Mild liver disease & 17 & $2.0 \%$ \\
\hline Diabetes without complications & 22 & $2.6 \%$ \\
\hline Diabetes with complications & 88 & $10.6 \%$ \\
\hline Paraplegia and hemiplegia & 12 & $1.4 \%$ \\
\hline Renal Disease & 52 & $6.3 \%$ \\
\hline Moderate or severe liver disease & 51 & $6.1 \%$ \\
\hline \multicolumn{3}{|l|}{ Type of symptoms } \\
\hline Typical & 602 & $72.4 \%$ \\
\hline Atypical & 230 & $27.6 \%$ \\
\hline \multicolumn{3}{|l|}{ Duration of GERD (years) } \\
\hline$<1$ & 71 & $8.5 \%$ \\
\hline $1-3$ & 153 & $18.4 \%$ \\
\hline $4-7$ & 187 & $22.5 \%$ \\
\hline $8-11$ & 160 & $19.2 \%$ \\
\hline $12-15$ & 80 & $9.6 \%$ \\
\hline $15+$ & 181 & $21.8 \%$ \\
\hline \multicolumn{3}{|l|}{$\begin{array}{l}\text { Duration Medication Use (PPI/H2/Other/Combo) } \\
\text { (years) }\end{array}$} \\
\hline$<1$ & $53 / 28 / 2 / 7$ & $6.4 / 3.4 / 0.2 / 0.8 \%$ \\
\hline $1-3$ & $91 / 21 / 2 / 20$ & $10.9 / 2.5 / 0.2 / 2.4 \%$ \\
\hline $4-7$ & $100 / 19 / 4 / 22$ & $12.0 / 2.3 / 0.5 / 2.6 \%$ \\
\hline $8-11$ & $96 / 10 / 2 / 19$ & $11.5 / 1.2 / 0.2 / 2.3 \%$ \\
\hline $12-15$ & $50 / 5 / 3 / 15$ & 6.0/0.6/0.4/1.8\% \\
\hline $15+$ & $124 / 11 / 9 / 30$ & $14.9 / 1.3 / 1.1 / 3.6 \%$ \\
\hline \multicolumn{3}{|l|}{ Barrett's Esophagus } \\
\hline Short segment & 33 & $4.0 \%$ \\
\hline Long segment & 5 & $0.6 \%$ \\
\hline Dysplasia & 2 & $0.2 \%$ \\
\hline
\end{tabular}

that are larger than ours in terms of patient volumes, our HBC is capturing a larger share of the GERD patients due to our integrated care approach for these patients. 

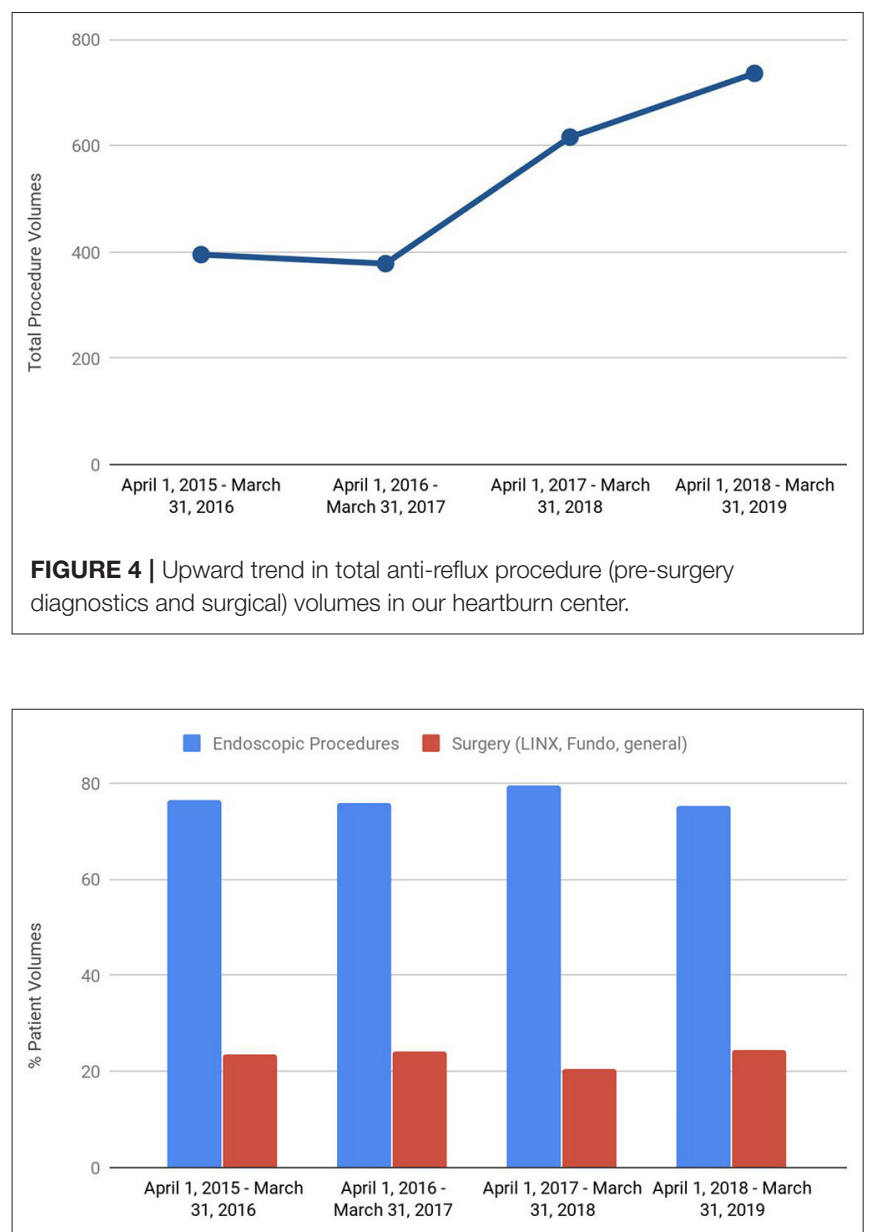

FIGURE 5 | Patient volumes as a percentage of the total pre-surgery diagnostics and surgical volumes.

\section{Patient Volumes}

As illustrated in Figure 4, there was an upward trend in patient volumes to the HBC year-over-year. Specifically, compared to pre- HBC, patient volumes increased by $75 \%$ post-HBC.

The uptick in volumes was across the board including pre-surgery diagnostics as well as anti-reflux surgical procedures. Importantly, an integrated approach provided the larger institution an opportunity to adopt novel surgical procedures such as $\operatorname{LINX}^{\circledR}$, as well as non-surgical endoscopic procedures such as Radiofrequency Ablation, Endoscopic Mucosal Resections that were not performed prior to the start of the HBC.

As depicted in Figure 5, even as the patient volumes have increased, the conversion rates from diagnostics to surgery have remained consistent in part due to our protocol-driven approach and management of the disease.

\section{Length of Stay}

The length of stays (LOS) for the two important anti-reflux procedures, LINX ${ }^{\circledR}$ and RTF, were recorded. As illustrated in Figure 6, as we gained more experience with these patients,

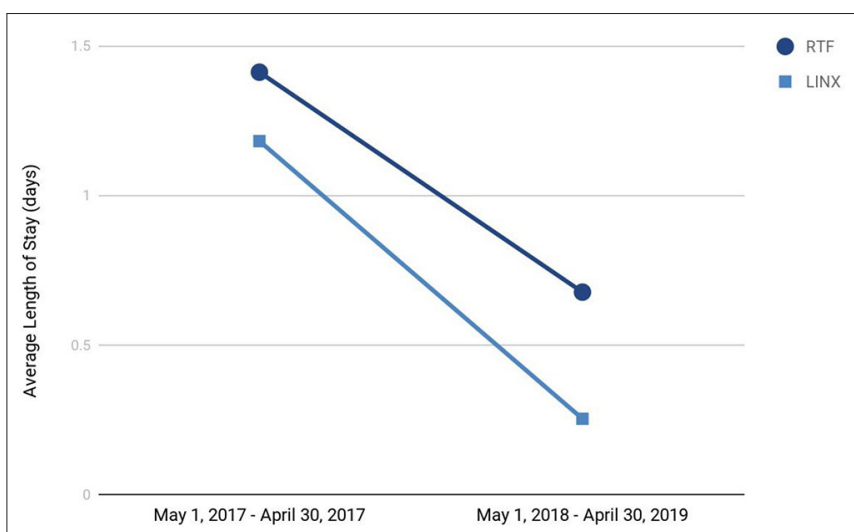

FIGURE 6 | Trends in average length of stay for the two primary anti-reflux surgeries conducted at our heartburn center.

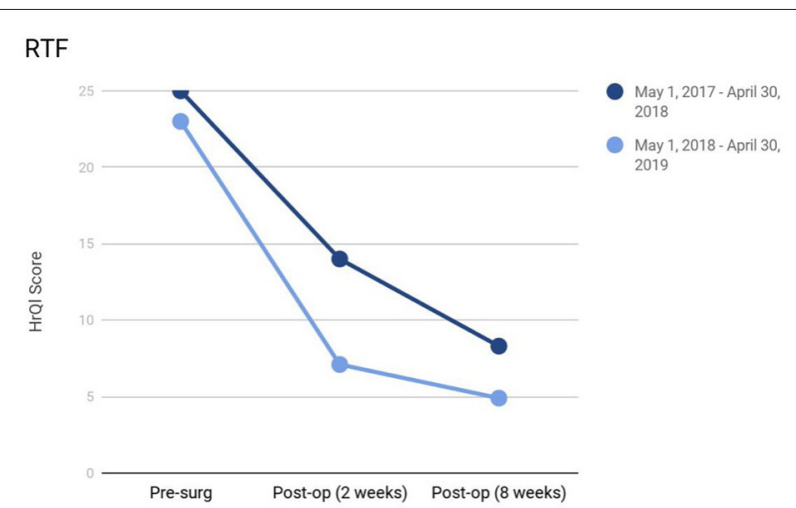

LINX

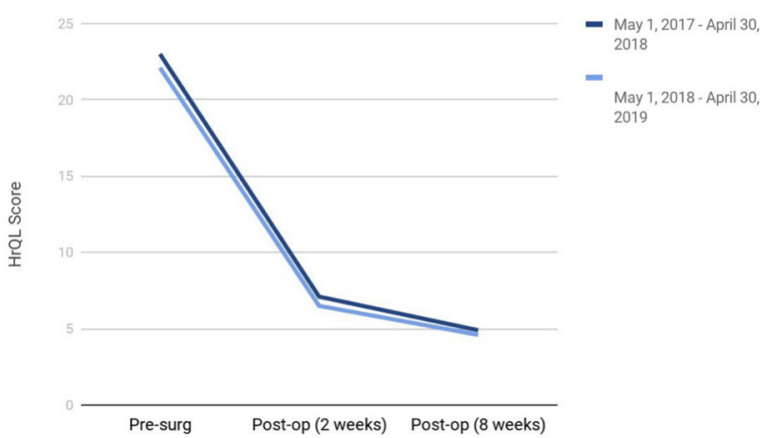

FIGURE 7 | Two-year trends in HRQL scores for patients undergoing LINX ${ }^{\circledR}$ and RTF.

the LOS reduced dramatically by over $50 \%$ over 2 years. The reduction in LOS resulted in significant economic benefit to the hospital.

\section{Health-Related Quality of Life (HRQL)}

Patients implanted with LINX ${ }^{\circledR}$ as well as those who underwent RTF demonstrated a statistically significant improvement in GERD-HRQL score at 8 weeks compared to baseline: 
An average score of 24 (range 36-0) vs. 6 (range 280 ) in LINX $^{\circledR}$ patients and 23 (range 41-0) vs. 5 (range 23-0) in the RTF population. As illustrated in Figure 7, while HRQL consistently improved post-surgery in both the populations, there was no significant difference in the HRQL improvement year-over-year.

\section{DISCUSSION}

An integrated GERD service line center offers a comprehensive, multi-specialty, and coordinated patient-centered approach. Ranging from initial consultation and diagnosis to surgical intervention for complex disease, such an approach is likely to provide optimal care and provide surveillance for patients for a complex disease process of GERD.

In this article, we reported our approach to a practice model of an integrated HBC based on our experience at our facility. The integrated approach drove quality and efficiency in terms of coordinated care that enabled care cross-over and efficient resource utilization. Furthermore, setting up treatment protocols and defining patient pathways standardized the workflow and provided a definition of the center. Importantly, local outreach and marketing helped develop interpersonal relationships with referral physicians resulting in recognition of the center as a "Real Antireflux Heartburn Center." That in turn promoted specialization and differentiation from competition, leading to superior results for our $\mathrm{HBC}$ both in terms of patient volumes and patient care. Following an audit of the retrospective data presented here, the regional Excellus BlueCros BlueShield now

\section{REFERENCES}

1. Gawron AJ, French DD, Pandolfino JE, Howden CW. Economic evaluations of gastroesophageal reflux disease medical management. Pharmacoeconomics. (2014) 32:745-58. doi: 10.1007/s40273-014-0164-8

2. El-Serag HB, Sweet S, Winchester CC, Dent J. Update on the epidemiology of gastro-oesophageal reflux disease: a systematic review. Gut. (2014) 63:871-80. doi: 10.1136/gutjnl-2012-304269

3. Toghanian S, Johnson DA, Stålhammar NO, Zerbib F. Burden of gastro-nnreflux disease in patients with persistent and intense symptoms despite proton pump inhibitor therapy: a post hoc analysis of the 2007 national health and wellness survey. Clin Drug Investig. (2011) 31:703-15. doi: 10.2165/11595480-000000000-00000

4. Kimura Y, Kamiya T, Senoo K, Tsuchida K, Hirano A, Kojima H, et al. Persistent reflux symptoms cause anxiety, depression, and mental health and sleep disorders in gastroesophageal reflux disease patients. J Clin Biochem Nutr. (2016) 59:71-7. doi: 10.3164/jcbn.16-9

5. Jung HK, Choung RS, Talley NJ. Gastroesophageal reflux disease and sleep disorders: evidence for a causal link and therapeutic implications. $J$ Neurogastroenterol Motil. (2010) 16:22-9. doi: 10.5056/jnm.2010.16.1.22

6. Lee YS, Jang BH, Ko SG, Chae Y. Comorbid risks of psychological disorders and gastroesophageal reflux disorder using the national health insurance service-National Sample Cohort: A STROBE-compliant article. Medicine. (2018) 97:e0153. doi: 10.1097/MD.0000000000010153

7. Chen $\mathrm{CH}$, Lin $\mathrm{CL}$, Kao CH. Association between gastroesophageal reflux disease and coronary heart disease: a nationwide populationbased analysis. Medicine. (2016) 95:e4089. doi: 10.1097/MD.0000000000 004089 considers the LINX ${ }^{\circledR}$ device implant a medically necessary procedure and provides insurance coverage to its 1.5 million members (26). Challenges remain to manage obese patients with GERD, the hospitals controlling their patients to be referred across the hospital systems, acceptance by the primary care and even some GI physicians to consider GERD to be a surgically manageable disease. The limitations of our study is that it is a single center effort and since we were not able to find other centers of similar integrity in a community set up, we were unable to compare our results with other centers. The community hospitals will need to establish similar set up to address disease of such complex nature to provide best care to the patients. Further discussions and dialogues are needed. We believe that the integrated approach not only provides optimal patient care and satisfaction, but also a unique opportunity for the institution to grow and adapt novel technologies.

\section{DATA AVAILABILITY STATEMENT}

The raw data supporting the conclusions of this article will be made available by the authors, without undue reservation.

\section{AUTHOR CONTRIBUTIONS}

All authors listed have made a substantial, direct and intellectual contribution to the work, and approved it for publication.

\section{ACKNOWLEDGMENTS}

Aniket Maini for proof reading and assisting in compiling the data in the paper.

8. Moraes-Filho JP, Navarro-Rodriguez T, Eisig JN, Barbuti RC, Chinzon D, Quigley EM. Comorbidities are frequent in patients with gastroesophageal reflux disease in a tertiary health care hospital. Clinics. (2009) 64:785-90. doi: 10.1590/\$1807-59322009000800013

9. Richter JE. Gastroesophageal reflux disease treatment: side effects and complications of fundoplication. Clin Gastroenterol Hepatol. (2013) 11:46571. doi: 10.1016/j.cgh.2012.12.006

10. Gikas A, Triantafillidis JK. The role of primary care physicians in early diagnosis and treatment of chronic gastrointestinal diseases. Int J Gen Med. (2014) 7:159-73. doi: 10.2147/IJGM.S58888

11. Badillo R, Francis D. Diagnosis and treatment of gastroesophageal reflux disease. World J Gastrointest Pharmacol Ther. (2014) 5:105-12. doi: 10.4292/wjgpt.v5.i3.105

12. Kinchen KS, Cooper LA, Levine D, Wang NY, Powe NR. Referral of patients to specialists: factors affecting choice of specialist by primary care physicians. Ann Fam Med. (2004) 2:245-52. doi: 10.1370/afm.68

13. Elrod JK, Fortenberry JL. Centers of excellence in healthcare institutions: what they are and how to assemble them BMC. Health Serv Res. (2017) 17:425. doi: 10.1186/s12913-017-2340-y

14. Robinson JC, MacPherson K. Payers test reference pricing and centers of excellence to steer patients to low-price and high-quality providers. Health Aff. (2012) 31:2028-36. doi: 10.1377/hlthaff.2011.1313

15. Scally CP, Shih T, Thumma JR, Dimick JB. Impact of a national bariatric surgery center of excellence program on medicare expenditures. J Gastrointest Surg. (2016) 20:708-14. doi: 10.1007/s11605-015-3 027-5

16. Mehrotra A, Sloss EM, Hussey PS, Adams JL, Lovejoy S, SooHoo NF. Evaluation of a center of excellence program for spine surgery. 
Med Care. (2013) 51:748-57. doi: 10.1097/MLR.0b013e31829 b091d

17. Rodak S. Is center of excellence investment the silver bullet healthcare has been looking for? (2013). Available online at: http://www.beckershospitalreview. com/hospital-key-specialties/is-center-of-excellence-investment-the-silverbullet-healthcare-has-been-looking-for.html

18. Kelly P, Chinta R, Privitera G. Do centers of excellence reduce health care costs? evidence from the us veterans health administration centers for epilepsy. Glob Bus Organization Excell. (2015) 34:18-29. doi: 10.1002/joe.21632

19. Yadlapati R, Gawron AJ, Bilimoria K, Keswani RN, Dunbar KB, Kahrilas PJ, et al. Development of quality measures for the care of patients with gastroesophageal reflux disease. Clin Gastroenterol Hepatol. (2015) 13:874-83. doi: 10.1016/j.cgh.2014.11.012

20. Schlottmann F, Strassle PD, Patti MG. Antireflux surgery in the USA: influence of surgical volume on perioperative outcomes and costs-time for centralization? World J Surg. (2018) 42:2183-9. doi: 10.1007/s00268-017-4429-1

21. Halpern R, Kothari S, Fuldeore M, Zarotsky V, Porter V, Dabbous $\mathrm{O}$, et al. GERD-related health care utilization, therapy, and reasons for transfer of GERD patients between primary care providers and gastroenterologists in a US managed care setting. Dig Dis Sci. (2010) 55:32837. doi: 10.1007/s10620-009-0927-9

22. Buckley FP, Havemann B, Chawla A. Magnetic sphincter augmentation: optimal patient selection and referral care pathways. World J Gastrointest Endosc. (2019) 11:472-6. doi: 10.4253/wjge.v11.i8.443

23. Kahrilas PJ, Shaheen NJ, Vaezi MF. American Gastroenterological Association Institute technical review on the management of gastroesophageal reflux disease. Gastroenterology. (2008) 135:1392-413. doi: 10.1053/j.gastro.2008.08.044

24. Katz PO, Gerson LB, Vela MF. Guidelines for the diagnosis and management of gastroesophageal reflux disease. Am J Gastroenterol. (2013) 108:308-28. doi: 10.1038/ajg.2012.444
25. Jobe BA, Richter JE, Hoppo T, Peters JH, Bell R, Dengler WC, et al. Preoperative diagnostic workup before antireflux surgery: an evidence and experience-based consensus of the esophageal diagnostic advisory panel. J Am Coll Surg. (2013) 217:586-97. doi: 10.1016/j.jamcollsurg.2013. 05.023

26. Blue Cross Blue Shield. Blue Cross Blue Shield Now Covers the LINX Procedure! (2020). Available online at: https://www.iersurgery.com/blog/bluecross-blue-shield-now- covers- the-linx-procedure/\#: :text=The\%20LINX \%20Reflux\%20Management\%20System,over\%2015\%20million\%20BCBS $\% 20$ members

27. Runge TM, Abrams JA, Shaheen NJ. Epidemiology of Barrett's Esophagus and Esophageal Adenocarcinoma. Gastroenterol Clin North Am. (2015) 44:203-31. doi: 10.1016/j.gtc.2015. 02.001

Conflict of Interest: The authors declare that the research was conducted in the absence of any commercial or financial relationships that could be construed as a potential conflict of interest.

Publisher's Note: All claims expressed in this article are solely those of the authors and do not necessarily represent those of their affiliated organizations, or those of the publisher, the editors and the reviewers. Any product that may be evaluated in this article, or claim that may be made by its manufacturer, is not guaranteed or endorsed by the publisher.

Copyright (๑) 2021 Maini, Sun, Buniak, Jantsch, Czajak, Frey, Kumar and Chawla. This is an open-access article distributed under the terms of the Creative Commons Attribution License (CC BY). The use, distribution or reproduction in other forums is permitted, provided the original author(s) and the copyright owner(s) are credited and that the original publication in this journal is cited, in accordance with accepted academic practice. No use, distribution or reproduction is permitted which does not comply with these terms. 